\title{
Factores sociodemográficos y nutricionales asociados a anemia en niños de 1 a 5 años en Perú
}

\section{Sociodemographic and nutritional factors associated with anemia in children aged 1 to 5 years old in Peru}

\section{RESUMEN}

Objetivo: Determinar los factores sociodemográficos y nutricionales asociados a la anemia en niños de 1 a 5 años de Perú según la Encuesta Nacional Demográfica y de Salud Familiar (DHS, por sus siglas en inglés Demographic and Health Survey). Metodología: Para determinar los posibles factores sociodemográficos y nutricionales asociados a la anemia, se hizo un subanálisis de la DHS 2017, tomando en cuenta un intervalo de confianza del $95 \%$ y considerando los parámetros de complejidad de la encuesta. Se describieron las frecuencias relativas en el análisis univariado. Para el análisis bivariado se empleó el test de chi cuadrado para las variables categóricas y t-Student para las variables continuas. Para el modelo de regresión múltiple, se realizó una regresión de Poisson cruda y ajustada con el método de selección de variables tipo backward. Principales resultados: La prevalencia de anemia fue de $38.5 \%$. Los factores independientemente asociados fueron quintil de riqueza bajo $(R P(\mathrm{a}): 1,23$; IC95\%: 1,0-1,4), ningún grado de instrucción o grado de instrucción primaria de la madre (RP(a): 1,25; IC95\%: 1,0-1,5), edad de la madre menor de 19 años (RP(a): 1,34; IC95\%: 1,1-1,7), lugar del parto no institucionalizado (RP(a): 1,24; IC95\%: 1,1-1,5), no consumo de antiparasitarios

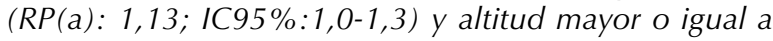
$4000 \mathrm{msnm}(R P(\mathrm{a}): 1,45$; IC95\%: 1,2-1,8). Conclusiones: La prevalencia de anemia en niños de 1 a 5 años en el 2017 fue moderada. Existen factores sociodemográficos y nutricionales asociados a dicha condición.

Palabras clave: Anemia; Encuestas epidemiológicas; $\mathrm{Nu}$ trición del niño; Perú; Salud del Niño.

\footnotetext{
ABSTRACT

Purpose: Determine the sociodemographic and nutritional factors associated with anemia in children aged 1 to 5 years in Peru according to the Demographic and Health Survey (DHS). Methods: To determine the possible sociodemographic and nutritional factors associated with anemia, a sub-analysis of the DHS 2017 was carried out, taking into account a 95\% confidence interval and considering the complexity of survey parameters. Relative
}

Ali Al-kassab-Córdova ${ }^{1,2}$, Carolina Méndez-Guerra ${ }^{1,2^{*}}$, Pamela Robles-Valcarcel ${ }^{3}$.

\begin{abstract}
1. Escuela de Medicina Humana, Universidad Peruana de Ciencias Aplicadas. Lima, Perú.

2. Sociedad Científica de Estudiantes de Medicina de la Universidad Peruana de Ciencias Aplicadas (SOCIEMUPC). Lima, Perú. 3. Programa de Nutrición y Dietética, Universidad Peruana de Ciencias Aplicadas. Lima, Perú.
\end{abstract}

*Dirigir Correspondencia: Carolina Isabel Méndez Guerra, Jirón Cristóbal de Peralta Sur 1241 Urb. Valle Hermoso; Surco; Lima, Perú Email: mendezguerra.ci@gmail.com

frequencies were described in univariate analysis. For bivariate analysis, the chi-square test was used for categorical variables and Student $t$-test for continuous variables. For the multivariable analysis, a crude and an adjusted Poisson regression was done with backward method for variable selection. Main results: Independently associated factors were low wealth (aPR: 1.23; 95\% Cl: 1.0-1.4), no degree or primary education level of the mother ( $a P R: 1.25$; 95\% Cl: 1.0-1.5), mother's age under 19 years (aPR: 1.34; 95\% Cl: 1.1-1.7), not delivering in a health facility (aPR: 1.24; 95\% Cl: 1.1-1.5), no consumption of antiparasitic drugs (aPR: 1.13; 95\% Cl: 1.0-1.3) and living at greater than or equal to 4000 meters above sea level (aPR: 1.45; $95 \% \mathrm{Cl}: 1.2-1.8)$. Conclusions: The prevalence of anemia 
in children aged 1 to 5 years in 2017 was moderate. There are sociodemographic and nutritional factors associated with this condition.

Keywords: Anemia; Child Health; Child Nutrition; Health Surveys; Peru.

\section{INTRODUCCIÓN}

La anemia es una condición médica de extensión mundial caracterizada por una baja concentración sanguínea de hemoglobina $(\mathrm{Hb})^{1}$. La Organización Mundial de la Salud (OMS) define anemia, en niños de 5 a 59 meses, como una concentración sanguínea de $\mathrm{Hb}$ menor a $11 \mathrm{~g} /$ dL. A nivel mundial, la OMS estima que afecta al $42,6 \%$ de niños en edad preescolar ${ }^{2}$. En Perú, la prevalencia de anemia en niños menores de 3 años durante el 2007 al 2013 alcanzó el $47,9 \%{ }^{3}$. La anemia se atribuye en un $50 \%$ al consumo deficitario de hierro, por lo cual se estima que el $42 \%$ de casos de anemia en niños mejoraría con la suplementación de dicho micronutriente ${ }^{5,6}$. Asimismo, una de las principales consecuencias de la anemia es la desnutrición crónica infantil, la cual afecta al $13,1 \%$ de niños peruanos menores de 5 años ${ }^{7}$. Esta se asocia a defectos en el desarrollo cerebral, cognitivo, motor y conductual ${ }^{8}$.

Diversos factores se han visto asociados al desarrollo de anemia en la niñez, entre los cuales resaltan las deficiencias nutricionales, infecciones parasitarias, bajos ingresos familiares, lactancia materna exclusiva deficitaria, bajo nivel educativo de la madre alcanzado, entre otros. Un estudio realizado en niños en edad escolar halló que las deficiencias nutricionales e infecciones parasitarias intestinales son factores predictores de anemia por deficiencia de hierro (IDA, por sus siglas en inglés Iron Deficiency Anemia). Se estimó que la ingesta inadecuada de productos proteicos y lácteos incrementan en 2,3 y 1,8 veces la probabilidad de anemia, respectivamente ${ }^{4}$. Igualmente, el consumo deficitario de huevo, vegetales y frutas se ha visto asociado a anemia en niños menores de 5 años ${ }^{9}$. Del mismo modo, la introducción tardía de frutas y verduras en la alimentación complementaría del lactante incrementa la frecuencia de anemia ${ }^{10}$. Un metaanálisis realizado por Iglesias et a ${ }^{11}$ describió que las intervenciones nutricionales reducen la anemia en un 25 a $44 \%$ en países latinoamericanos. También se ha descrito que las infecciones parasitarias incrementan el riesgo de anemia en 1,45 veces ${ }^{4}$. Inclusive, se ha determinado que la ausencia de una terapia preventiva antiparasitaria en niños menores de 3 años se asocia a anemia ${ }^{3}$. Otros factores asociados a anemia son madres adolescentes, bajo nivel educativo alcanzado y bajo nivel socioeconómico ${ }^{3}$. En contraparte, la lactancia materna exclusiva previene el desarrollo de anemia en los primeros meses de vida ${ }^{12}$.

El presente estudio busca determinar los factores sociodemográficos y nutricionales asociados a la anemia en niños de 1 a 5 años mediante un subanálisis de la Encuesta Demográfica y de Salud Familiar ((DHS, por sus siglas en inglés Demographic and Health Survey) del año 2017.

\section{MATERIALES Y MÉTODOS \\ Diseño y población de estudio}

Se realizó un estudio observacional transversal analítico a partir de la Encuesta Demográfica y de Salud Familiar (DHS) del año 2017. La población de estudio incluyó niños de 1 a 5 años participantes de la DHS del 2017 quienes contaban con una medición de hemoglobina sanguínea ajustada por altitud. El tamaño muestral de la DHS 2017 fue de 35.910 viviendas. La base de datos se encuentra disponible en la sección Microdatos del portal web del Instituto Nacional de Estadística e Informática (INEI), a la cual se puede acceder a través del siguiente enlace: http:// iinei.inei.gob.pe/microdatos/.

\section{Fuente de datos}

La DHS es una importante investigación estadística, de carácter continuo, realizada por el INEI. Contiene información actualizada sobre la salud reproductiva y salud materno-infantil; por lo cual permite analizar tendencias y cambios relacionados a dichos tópicos en infantes y mujeres en edad reproductiva. El marco muestral estuvo constituido por la información estadística y cartográfica actualizada proveniente de los Censos Nacionales XI de Población y VI de Vivienda del 2007 y la Actualización SISFOH 20122013. Las unidades de muestreo estuvieron constituidas por el conglomerado y vivienda particular en el área urbana, y el área de empadronamiento rural y vivienda particular en el área rural. El muestreo fue bietápico, probabilístico de tipo equilibrado, estratificada e independiente a nivel departamental y por área urbana y rural.

\section{Medición de la hemoglobina sanguínea}

La concentración de hemoglobina en la DHS fue medida con un hemoglobinómetro portátil (HemoCue ${ }^{\circledR}$ ) basado en el método de Vanzetti. Este método se basa en la utilización de microcubetas de pequeño volumen (10 $\mu \mathrm{L}$ ) que contienen deoxicolato de sodio, nitrito de sodio y azida de sodio. La muestra de sangre capilar se obtiene del dedo anular o medio de la mano. Dicha muestra se vierte, por capilaridad, en la microcubeta donde se mezcla espontáneamente con el reactivo previamente descrito. En la microcubeta se lleva a cabo una modificación de la reacción de la azidametahemoglobina. Finalmente, la microcubeta es leída en un hemoglobinómetro a una absorbancia de $565 \mathrm{~nm}$ y $880 \mathrm{~nm}^{9}$. El resultado se muestra en forma digital. La sensibilidad del presente método es del $100 \%$ y la especificidad del $94 \%$. ${ }^{3,13}$. El ajuste por altitud se basó en la fórmula establecida por el Pediatric Nutrition Surveillance System (CDCPNSS) ${ }^{13}$.

\section{Variables de estudio}

La variable dependiente (anemia) se definió, según la OMS, como la concentración de hemoglobina $<11$ g/ $\mathrm{dL}$ para niños entre 1 y 5 años $^{14}$. El valor de hemoglobina obtenido fue corregido por la altitud en la que se encontraba el participante. Las variables independientes se clasificaron 
como factores sociodemográficos y nutricionales. Los factores sociodemográficos seleccionados fueron: edad del niño, sexo del niño, ámbito de la vivienda, región, altitud, quintil de riqueza, lugar del parto, edad materna y grado de instrucción de la madre. Los factores nutricionales seleccionados fueron: consumo de leche; consumo de queso, yogurt, otros productos lácteos; consumo de calorías discrecionales; consumo de frijoles, arverjas y nueces; consumo de hortalizas de hoja verde; consumo de frutas secas; el niño recibe suplementos de hierro; madre recibió suplementos de hierro durante el embarazo; recibe suplementos de vitamina $A$; tiempo de lactancia materna; y consumo de antiparasitarios por parte del niño en los últimos 6 meses.

\section{Procesamiento y análisis estadístico de datos}

La base de datos fue diseñada en el programa RStudio ${ }^{\circledR}$ donde se seleccionaron las variables de interés a partir de los módulos 66 (datos básicos), 67 (historia de nacimientotabla de conocimiento de método), 69 (embarazo, parto, puerperio y lactancia), 70 (inmunización y salud) y 74 (peso y talla-anemia). La base de datos fue exportada en formato DTA.

El análisis de datos se realizó en el programa Stata ${ }^{\circledR}$ versión 14.0 (Stata Corporation, College Station, TX, USA). Se utilizó el módulo de comandos svy para encuestas complejas. Para seleccionar la población de niños de 1 a 5 años se creó una subpoblación. Para el análisis univariado se describieron las frecuencias relativas de las variables categóricas y las medidas de tendencia central y dispersión de las variables continuas. Para el análisis bivariado, al comparar variables categóricas se utilizó la prueba de chi cuadrado. Al comparar variables categóricas y continuas se empleó la prueba t-Student. Para el modelo de regresión múltiple, se hizo una regresión de Poisson, empleando el método de selección de variables tipo backward. Finalmente se reportaron las razones de prevalencia (RP) con sus valores $p$ respectivos. El análisis se hizo considerando los procesos de estimación para muestreo por conglomerados complejos multietápicos, tomando en cuenta las probabilidades de muestreo y las ponderaciones de la muestra.

\section{RESULTADOS}

La base de datos diseñada a partir de la DHS 2017 contó con 21.528 registros de niños menores de 5 años. Se realizó una subpoblación de niños de 1 a 5 años dando un total de 16.957 registros. De estos, 2.237 no contaron con la cuantificación de hemoglobina; por lo cual, nuestra población de estudio fue de 14.720 niños.

Los factores sociodemográficos son descritos en la primera columna de la tabla 1 . La edad media fue de 36,5 meses. De la población estudiada, el 50,8\% eran mujeres, el 60,8\% vivían en zona urbana y el 38,9\% pertenecía al 1er quintil (muy pobre). Con respecto a las madres, el $97,6 \%$ eran mayores de 19 años y el $54,2 \%$ no tenían educación o contaban con educación primaria (Tabla 1).
Los factores nutricionales son descritos en la primera columna de la tabla 2. Con respecto a los productos derivados del ganado vacuno, el $61,7 \%$ reportaron no consumir leche $y$, de manera similar, el $71,9 \%$ negaron el consumo de queso, yogurt u otros productos lácteos. Asimismo, el 62,9\% afirmó consumir productos cárnicos y el 20,4\% reportó el consumo de calorías discrecionales. Con respecto a la suplementación de hierro, el 90,2\% de madres reportaron haberla recibido durante el embarazo mientras que el $40,0 \%$ de niños reportó recibirla al momento de la entrevista. Otros hallazgos a resaltar son que el $71,9 \%$ recibió lactancia materna durante 6 meses o más y el 32,6\% reporta haber recibido antiparasitarios en los últimos 6 meses (Tabla 2).

La prevalencia de anemia fue del $38,5 \%$; de la cual, en su mayoría, se clasificaba como anemia leve. La media de hemoglobina ajustada por altitud fue de $11,2 \pm 1,3 \mathrm{~g} / \mathrm{dL}$. La edad donde se concentró la mayor frecuencia de anemia fue a los 35 meses. Las variables sociodemográficas cuya asociación fue estadísticamente significativa fueron ámbito de la vivienda $(p<0,01)$, región $(p<0,01)$, altitud $(p<0,01)$, quintil de riqueza $(p<0,01)$, lugar del parto $(p<0,01)$, edad de la madre $(p<0,01)$, grado de instrucción de la madre $(p<0.01)$ y la edad del niño $(p<0,01)$. Por otro lado, las variables nutricionales cuya asociación fue estadísticamente significativa fueron consumo de queso, yogurt, u otros productos lácteos $(p=0,04)$; consumo de hortalizas de hoja verde $(p<0,01)$; consumo de suplementos de hierro por parte del niño $(p=0,04)$ y de la madre durante el embarazo $(p=0,03)$; y tomar antiparasitarios $(p=0,04)$ (Tablas 1 y 2 ).

En el modelo de regresión múltiple crudo se analizaron 12 variables. En el modelo de regresión múltiple ajustado se analizaron solo 6 variables, previo descarte de las restantes a través del método de selección backward. Aquellos sujetos de estudio que viven a más de 4.000 msnm resultaron tener 1,45 veces más probabilidades de sufrir de anemia $(P R=1,45, p<0.01, I C=1,2-1,8)$. Mientras que vivir por debajo de 3000 msnm resultó ser un factor protector. El quintil de riqueza categorizada como pobre resultó tener 1,23 veces más probabilidades de sufrir de anemia $(P R(a)=1,23, p=0.01, I C=1,0-1,4)$. Mientras que en quintil de riqueza rico y muy rico resultó ser un factor protector. Por otra parte, las madres menores de 19 años tuvieron 1,34 veces más probabilidades que sus hijos padecieran de anemia $(\mathrm{PR}(\mathrm{a})=1,34, \mathrm{p}<0,01, \mathrm{IC}=1,1$ $1,7)$. Aquellos niños hijos de madres que no contaban con estudios o tenían educación primaria, resultaron tener 1,25 veces más probabilidades de tener anemia $(\mathrm{PR}(\mathrm{a})=1,25, \mathrm{p}=0,01, \mathrm{IC}=1,0-1,5)$. Aquellos niños quienes sus madres tuvieron un parto no institucionalizado tuvieron 1,24 veces más probabilidades de tener anemia $(P R(a)=1,24, p<0.01, I C=1,1-1,5)$. Los niños que no tomaron antiparasitarios resultaron tener 1,13 más probabilidades de padecer de anemia $(\operatorname{PR}(\mathrm{a})=1,13, \mathrm{p}=$ $0,02, \mathrm{IC}=1,0-1,3$ ) (Tabla 3). 
Tabla 1. Factores sociodemográficos asociados a an emia en niños de 1 a 5 años.

\begin{tabular}{|c|c|c|c|c|c|c|}
\hline \multirow{2}{*}{$\begin{array}{c}\text { Sexo } \\
\end{array}$} & \multirow[b]{2}{*}{ Hombre } & \multirow{2}{*}{$\begin{array}{c}\text { Población* } \\
\text { (\%) }\end{array}$} & \multirow{2}{*}{$\begin{array}{c}\begin{array}{c}\text { Anemia }^{\dagger} \\
(\%)\end{array} \\
49,0\end{array}$} & \multirow{2}{*}{$\begin{array}{c}\mathbf{P} \\
0,87\end{array}$} & \multicolumn{2}{|c|}{ IC 95\% } \\
\hline & & & & & 47,1 & 50,8 \\
\hline & Mujer & 50,8 & 51,0 & & 49,1 & 52,9 \\
\hline \multirow[t]{2}{*}{ Ámbito de la vivienda } & Urbana & 60,8 & 55,4 & $<0,01$ & 49,9 & 60,9 \\
\hline & Rural & 39,3 & 44,6 & & 39,1 & 50,1 \\
\hline \multirow[t]{4}{*}{ Región } & Lima & 22,9 & 17,8 & $<0,01$ & 14,1 & 22,2 \\
\hline & Resto costa & 24,9 & 21,1 & & 17,6 & 25,2 \\
\hline & Sierra & 26,9 & 29,9 & & 25,4 & 24,7 \\
\hline & Selva & 25,2 & 31,2 & & 25,6 & 37,5 \\
\hline Altitud & $<1000$ & 67,3 & 65,9 & $<0,01$ & 60,9 & 70,6 \\
\hline \multirow[t]{4}{*}{ (msnm) } & 1000-1999 & 5,6 & 3,8 & & 2,6 & 7,7 \\
\hline & 2000-2999 & 10,7 & 9,2 & & 6,6 & 12,8 \\
\hline & 3000-3999 & 15,3 & 19,0 & & 15,6 & 23,0 \\
\hline & $\geq 4000$ & 1,3 & 2,1 & & 1,1 & 3,8 \\
\hline \multirow[t]{5}{*}{ Quintil de riqueza } & Muy pobre (Q1) & 38,9 & 46,5 & $<0,01$ & 41,2 & 51,9 \\
\hline & Pobre (Q2) & 24,1 & 26,0 & & 22,0 & 32,4 \\
\hline & Medio (Q3) & 14,8 & 13,9 & & 11,5 & 16,7 \\
\hline & Rico (Q4) & 13,8 & 9,1 & & 7,2 & 11,4 \\
\hline & Muy rico (Q5) & 8,4 & 4,5 & & 3,3 & 6,1 \\
\hline \multirow[t]{2}{*}{ Lugar parto } & En institución & 80,2 & 74,3 & $<0,01$ & 68,3 & 79,3 \\
\hline & No en institución & 19,8 & 25,7 & & 20,6 & 31,6 \\
\hline \multirow[t]{2}{*}{ Edad de la madre (años) } & $<19$ & 2,4 & 0,8 & $<0,01$ & 0,5 & 1,3 \\
\hline & $\geq 19$ & 97,6 & 99,2 & & 98,7 & 99,5 \\
\hline Grado de instrucción & Ninguno/primaria & 54,2 & 63,0 & $<0,01$ & 58,5 & 67,4 \\
\hline \multirow[t]{2}{*}{ de la madre } & Secundaria & 25,6 & 23,6 & & 19,9 & 27,6 \\
\hline & Superior & 20,1 & 13,4 & & 11,4 & 15,9 \\
\hline Edad del niño (meses) & Media $\pm \mathrm{EE}^{++}$ & $36,5 \pm 0,2$ & $35,5 \pm 0,3$ & $<0,01$ & 34,5 & 35,8 \\
\hline 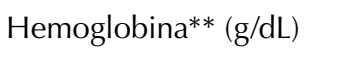 & Media \pm EE & $11,2 \pm 1,3$ & & & & \\
\hline \multirow[t]{2}{*}{ Anemia } & Sí & 38,5 & & & & \\
\hline & No & 61,5 & & & & \\
\hline \multirow{4}{*}{$\begin{array}{l}\text { Nivel de severidad de } \\
\text { anemia }^{++\dagger}\end{array}$} & Sin anemia & 61,5 & & & & \\
\hline & Leve & 26,2 & & & & \\
\hline & Moderado & 12,1 & & & & \\
\hline & Severo & 0,3 & & & & \\
\hline
\end{tabular}

*Análisis univariado; ** $\mathrm{Hb}$ ajustada por altitud; ${ }^{\dagger}$ Análisis bivariado. Se empleó la prueba de chi cuadrado para variables categóricas y la prueba t-Student para las variables continuas; ${ }^{+\dagger}$ Error estándar; ${ }^{++}$Se utilizó la clasificación de severidad de anemia de la OMS ${ }^{14}$. 
Tabla 2. Factores nutricionales asociados a anemia en niños de 1 a 5 año.

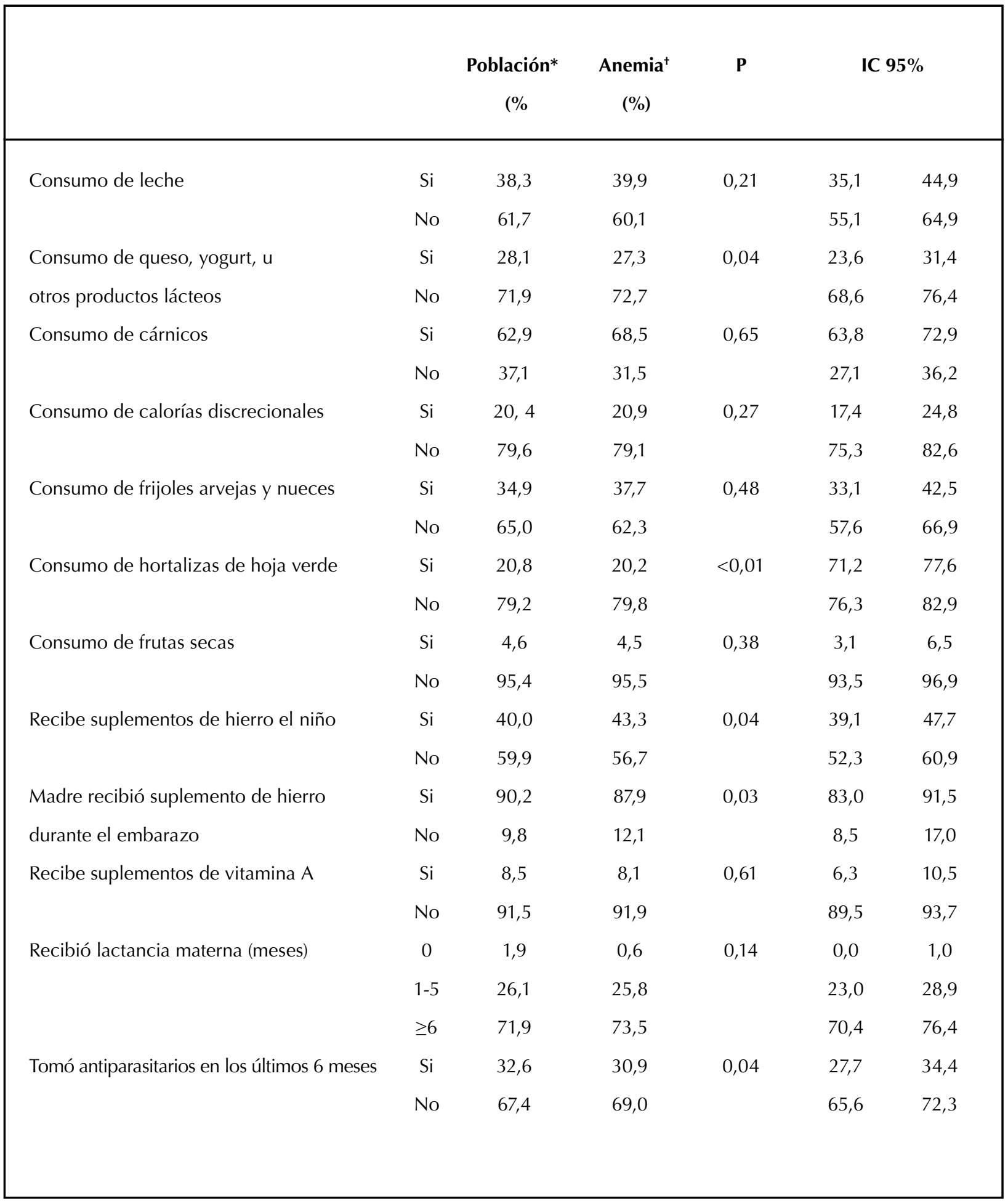

*Análisis univariado.

+ Análisis bivariado. Se empleó la prueba de chi cuadrado. 
Tabla 3. Modelo de regresión múltiple: factores asociados a la anemia en niños de 1 a 5 años.

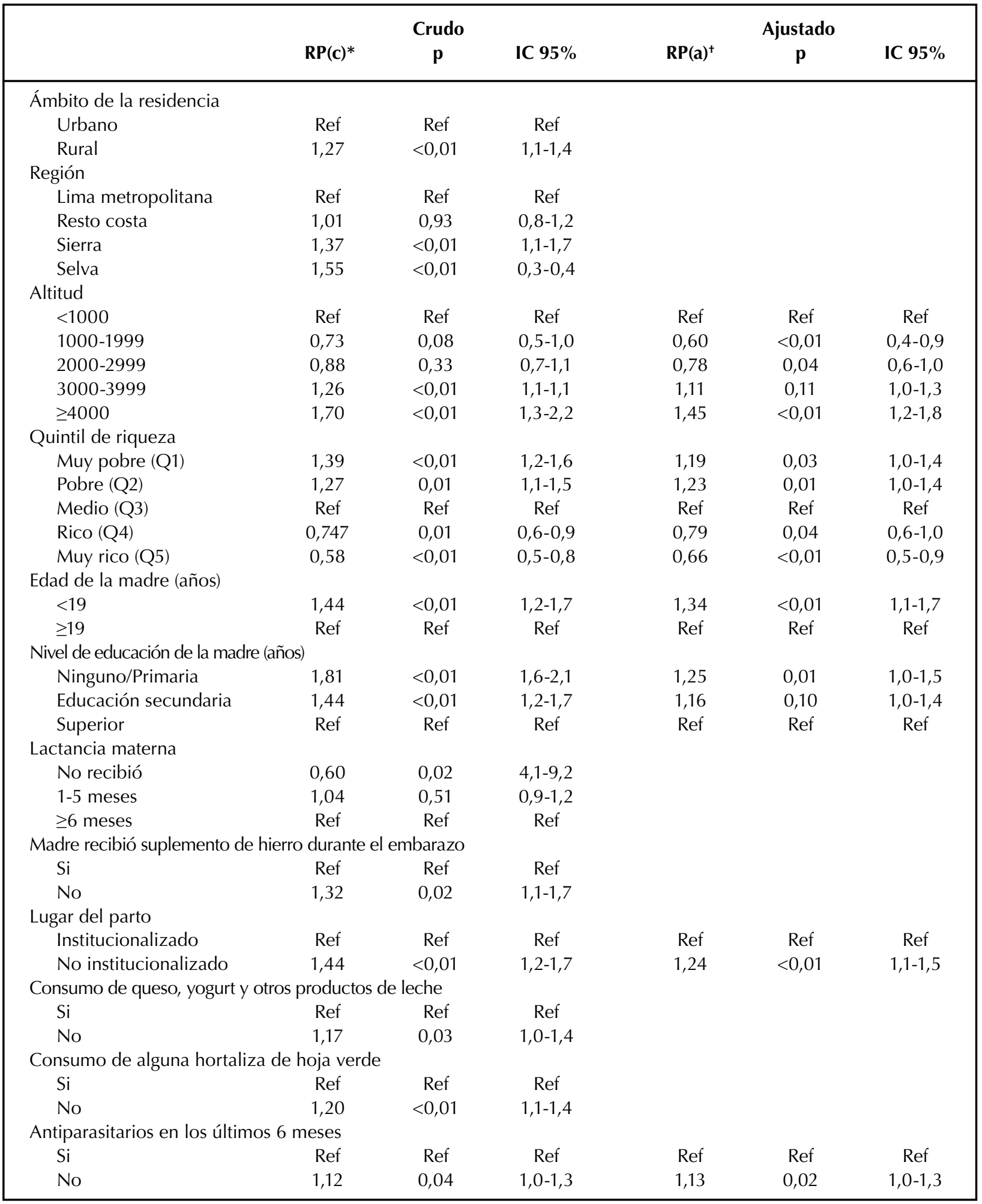

*RP(c): Razón de prevalencias cruda. ${ }^{+} \mathrm{RP}(\mathrm{a})$ : Razón de prevalencias ajustada. 


\section{DISCUSIÓN}

La prevalencia de anemia en niños de 1 a 5 años fue del $38,5 \%$ con una media de hemoglobina ajustada por altitud de 11,2 g/dL. Un estudio realizado en Perú que analizó la DHS, durante el 2007 al 2013, halló que la prevalencia de anemia en niños menores de 3 años fue del $47.9 \%{ }^{3}$. Dicha elevada frecuencia de anemia en niños menores de tres años es congruente con lo hallado en el presente estudio, lo cual indica que la mayor frecuencia de anemia se concentró en la edad de 35 meses. Un estudio realizado en el 2006 en La Paz, Bolivia encontró que la prevalencia de anemia en 114 niños de 6 a 24 meses, concurrentes a tres centros de salud, fue del $86 \%{ }^{15}$. De manera similar, un estudio realizado en el 2014 en Brasil halló que la prevalencia de anemia en 373 niños de 6 a 72 meses, concurrentes a 18 guarderías, fue de $38,3 \%{ }^{16}$. De tal manera, la OMS estima que Perú, Brasil y Bolivia son los países latinoamericanos con una las mayores cifras de anemia. Ante tal situación, el Ministerio de Salud (MINSA) formuló el Plan Nacional para la Reducción y Control de la Anemia Materno Infantil y la Desnutrición Crónica Infantil en el Perú: 2017-2021. Este espera reducir la tasa de anemia en niñas y niños de 6 a 36 meses al $19 \%$ para el $2021^{7}$.

Los quintiles de riqueza pobre y muy pobre resultaron tener $23 \%$ y $19 \%$ más probabilidad de padecer anemia que la clase media, respectivamente. Asimismo, pertenecer a un nivel socioeconómico rico o muy rico resultaron ser factores protectores. Dichos datos son similares a los encontrados en investigaciones conducidas en otros países. Un estudio realizado en China halló que los niños provenientes de familias con bajos ingresos tenían 14\% más riesgo de padecer anemia que niños cuyos ingresos familiares fueran altos ${ }^{17}$. Los hijos de madres menores de 19 años y madres con un bajo grado de instrucción tuvieron $33 \%$ y $25 \%$ más probabilidad de padecer anemia, respectivamente. Un estudio realizado en Perú durante el 2007 al 2013 halló que la anemia en niños menores de tres años era $30 \%$ más probable cuando la madre tenía menos de 19 años y $40 \%$ más frecuente cuando la madre tenía un grado de instrucción primario o nulo ${ }^{3}$. Asimismo, un estudio reportó que los hijos de madres con mayores niveles educativos consumían más proteínas y hierro de fuentes animales ${ }^{18}$. De tal forma, la baja educación materna supone un riesgo incrementado de padecer anemia en hogares de bajos recursos ${ }^{19}$. Por otra parte, vivir a menos de $2.000 \mathrm{msnm}$ tuvo un efecto protector, mientras que vivir por encima de los $4.000 \mathrm{msnm}$ incrementó en $45 \%$ la probabilidad de padecer anemia. Del mismo modo, un estudio realizado en Perú durante el 2007 al 2013 halló aquellos niños que vivían por encima de $4.000 \mathrm{msnm}$ tenían 3,7 veces más probabilidad de padecer anemia que quienes residían a menos de $1.000 \mathrm{msnm}$. Cabe resaltar que dicho estudio utilizó el OR, mientras que nosotros usamos $\mathrm{PR}^{3}$.

A diferencia de otros estudios ${ }^{20,21}$, en este estudio se encontró que quienes consumían queso, yogurt u otros productos lácteos, tenían menor prevalencia de anemia frente a quienes no consumían. Así, el 72,7\% de niños que no consumían productos derivados del ganado vacuno tenían anemia. Una revisión sistemática reportó que la fortificación de la leche con multimicronutrientes de hierro constituye una medida efectiva en la prevención de la anemia en niños que habitan en países en desarrollo. Esta intervención ha demostrado aumentar el nivel de hemoglobina en $0,87 \mathrm{~g} / \mathrm{dL}$ y reducir la anemia en un $57 \% \%^{22}$. Por otra parte, la prevalencia de anemia fue menor en aquellas personas que consumían hortalizas de hoja verde en comparación con las que no consumían. Así, solo el 20,2\% que consumía dicho alimento tenía anemia. Las hortalizas de hoja verde se caracterizan por tener un alto nivel de hierro ${ }^{23}$. Los datos obtenidos en cuanto a suplementación de hierro son incongruentes. Pese a la alta prevalencia de niños y madres que reciben suplementos de hierro, la prevalencia de anemia es alta. Esto podría explicarse por la baja adherencia al tratamiento preventivo y/o terapéutico ${ }^{24,25}$. Por otro lado, aquellos sujetos de estudio que reportaron no haber recibido antiparasitarios tuvieron $13 \%$ más probabilidad de padecer anemia en comparación con quienes si recibieron antiparasitarios en los últimos 6 meses. Dicho hallazgo se correlaciona con los resultados de un estudio realizado en Angola, el cual reportó que el $42,8 \%$ de niños anémicos sufrían, también, de una parasitosis ${ }^{26}$.

La principal limitación del presente estudio fue que no se contó con un estricto control de calidad de los datos registrados por tratarse de un análisis de base secundaria. Asimismo, hubiese sido de suma importancia contar con variables que no se encontraron en la base de datos, tales como la frecuencia del consumo de los alimentos. El instrumento de medición de hemoglobina fue adecuado y hace que los datos de este trabajo sean más fidedignos. No obstante, no se contó con la determinación de ferritina sérica, por lo cual, no se pudo especificar si la anemia era por deficiencia de hierro o por otra causa. Los datos del presente estudio son comparables a los de otros países puesto que, por el diseño de la DHS, se tienen datos estadísticos que reflejan la realidad del Perú.

Se recomienda que en próximos estudios se mida la frecuencia del consumo de los alimentos y se especifique el tipo de anemia a través de medición de ferritina sérica. Igualmente, se recomienda a la comunidad científica llevar a cabo estudios prospectivos en los cuales se cuente con la frecuencia de consumo de ciertos alimentos para determinar su posible efecto protector en la anemia. Asimismo, recomendamos que las industrias de productos lácteos fortifiquen los productos lácteos con hierro ya que dicha intervención ha demostrado disminuir la anemia de manera significativa. De la misma forma, los datos del presente estudio sirven para el diseño, implementación y redireccionamiento de planes de salud pública destinados a reducir la anemia en el Perú. 


\section{CONCLUSIONES}

La prevalencia de anemia en niños de 1 a 5 años durante el 2017 fue del 38,5\%. Se halló una asociación significativa entre ciertos factores sociodemográficos como el ámbito de residencia, región, altitud, quintil de riqueza, edad y grado de instrucción de la madre, y tipo de parto.

\section{BIBLIOGRAFÍA}

1. Lin TF, Huang IN, Cash HL. Comprehensive review of preschool age anemia in the pacific island jurisdictions. Hawaii / Med Public Health. 2017; 76: 331-336.

2. Cappellini MD, Motta I. Anemia in clinical practice-definition and classification: Does hemoglobin change with aging?. Semin Hematol. 2015; 52: 261-269.

3. Velásquez-Hurtado JE, Rodríguez Y, Gonzáles M, AsteteRobilliard L, Loyola-Romaní J, Vigo WE, et al. Factors associated with anemia in children under three years of age in perú: Analysis of the encuesta demográfica y de salud familiar, endes, 2007-2013. Biomedica 2016; 36: 220-229.

4. Desalegn Wolide A, Mossie A, Gedefaw L. Nutritional iron deficiency anemia: Magnitude and its predictors among school age children, southwest ethiopia: a community based cross-sectional study. PLoS One. 2014; 9: e114059.

5. Soundarya N, Suganthi P. A review on anaemia-types, causes, symptoms and their treatments. I Sci Technol Investig. 2016; 1: $10-17$.

6. World Health Organization. The global prevalence of anaemia in 2011 [Internet]. 2015. [Consulted Nov 15 2019]. Available from: https://www.who.int/nutrition/publications/ micronutrients/global_prevalence_anaemia_2011/en/

7. Ministerio de Salud. National plan for the reduction and control of maternal and childhood anemia and chronic child malnutrition in Peru: 2017-2021. Lima, 2017.

8. Zavaleta N, Astete-Robilliard L. Effect of anemia on child development: long-term consequences. Rev Peru Med Exp Salud Publica. 2017; 34: 716-722.

9. Pita-Rodríguez G, Jiménez-Acosta S, Basabe-Tuero B, Macías Matos C, Selva Suárez L, Hernández Fernández C, et al. The low consumption of iron-containing foods and enhacer of iron absorption are associated with anemia in preschool children of the eastern provinces of cuba: 2015-2011. Rev Chil Nutr. 2013; 40: 224-234.

10. Da Silva LLS, Fawzi WW, Cardoso MA. Factors associated with anemia in young children in brazil. PLoS One. 2018; 13: e0204504.

11. Iglesias Vázquez $L$, Valera $E$, Villalobos $M$, Tous $M$, Arija $V$. Prevalence of anemia in children from latin america and the caribbean and effectiveness of nutritional interventions: systematic review and meta-analysis. Nutrients. 2019; 11: 183.
12. Marques RF, Taddei JA, Lopez FA, Braga JA. Breastfeeding exclusively and iron deficiency anemia during the first 6 months of age. Rev Assoc Med Bras. 2014; 60: 18-22.

13. Instituto Nacional de Estadistica e Informatica. Anemia measurement methodology. Lima; 2018.

14. World Heath Organization. Hemoglobin concentrations for the diagnosis of anaemia and assessment of severity [Internet]. Ginebra; 2011 [consulted Dec 18 2019]. Available from: https://www.who.int/vmnis/indicators/haemoglobin/en/

15. Urdiqui C, Vera C, Trujillo N, Mejía H. Prevalence of anemia in children 6-24 months. Rev Chil Pediatr. 2008; 19: 327-331.

16. Chaves de Olivera TS, Costa Da Silva M, Nunes Santos J, Silva Rocha D, Llindgren Alves CR, Diniz Capanema F, et al. Anemia among pre-school children - a public health problem in belo horizonte, brazil. Cienc Saude Colet. 2014; 19: 59-66.

17. Xin QQ, Chen BW, Yin DL, Xiao F, Li RL, Yin T, et al. Prevalence of anemia and its risk factors among children under 36 months old in China. J Trop Pediatr. 2017; 63: 36-42.

18. Choi HJ, Lee HJ, Jang $H B$, Park $J Y$, Kang $J H$, Park $K H$, et al. Effects of maternal education on diet, anemia, and iron deficiency in korean school-aged children. BMC Public Health. 2011; 11: 870 .

19. Metallinos-Katsaras E, Colchamiro R, Edelstein S, Siu E. Household food security status is associated with anemia risk at age 18 months among low-income infants in Massachusetts. J Acad Nutr Diet. 2016; 116: 760-1766.

20. Levy-Costa RB, Monteiro CA. Cow's milk consumption and childhood anemia in the city of são Paulo, southern Brazil. Rev Saude Publica. 2004; 38: 797-803.

21. Oliveira MA, Osório MM. Cow's milk consumption and iron deficiency anemia in children. J Pediatr. 2005; 81: 361-367.

22. Eichle K, Wieser S, Rüthemann I, Brügger U, et al. Effects of micronutrient fortified milk and cereal food for infants and children: a systematic review. BMC Public Health. 2012; 12: 506.

23. Icard-Vernière C, Olive F, Picq C, Mouquet-Rivier C. Contribution of leafy vegetable sauces to dietary iron, zinc, vitamin A and energy requirements in children and their mothers in burkina faso. Plant Foods Hum Nutr. 2015; 70: 63-70.

24. Munares-García O, Gómez-Guizado G. Adherence to multimicronutrients and associated factors in children 6 to 35 months of sentinel sites, ministry of health, perú. Rev Bras Epidemiol. 2016; 19: 539-553.

25. Kiwanuka TS, Ononge S, Kiondo P, Namusoke F. Adherence to iron supplements among women receiving antenatal care at mulago national referral hospital, uganda-cross-sectional study. BMC Res Notes. 2017; 10: 510.

26. Oliveira D, Ferreira FS, Atouguia J, Fortes F, Guerra A, Centeno-Lima $S$. Infection by intestinal parasites, stunting and anemia in school-aged children from southern angola. PLoS One. 2015; 10: e0137327. 